\title{
Linear perspective as a cue in misperceived rotary motion
}

\author{
JOHN F. CROSS 1 \\ SAINT LOUIS UNIVERSITY
}

\begin{abstract}
Pairs of luminous lines of differing length ratios, rotated about an axis perpendicular to the line of sight, resulted in misperceptions of the direction of rotation. The illusions are similiar to the Ames trapezoid illusion. The results help to clarify the role of depth cues in visual perception of motion.
\end{abstract}

In 1951 Ames described a trapezoidal framework made to resemble a window sash viewed at an angle. When rotated on a vertical axis this framework appeared to oscillate with the taller side always nearer the viewer. This is the demonstration that Allport (1955) has called "a dramatic masterpiece of ambiguous stimulation." Ames explained the illusion as due to the fact that we have learned to interpret trapezoidal forms as rectangles. Doors, windows, walls, buildings, etc., most often cast a trapezoidal image on the retina, yet we perceive them as rectangular. In other words, we exhibit shape constancy. In the case of rectangles, the apparently taller side is always nearer, and therefore, with the rotating trapezoid, the taller side is seen as persistently nearer.

Recent work on this type of illusion, (Day \& Power, 1963; Mulholland, 1965; Murray, 1961; Pastore, 1952; Zegers, 1964) has directed attention to various factors affecting the illusion. The effects of viewing distance, size and shape of objects, tilt of axis, and surface pattern have been explored. There is fairly general agreement that the strength of the illusion increases with distance and decreases with object size and with tilt of axis toward the viewer. Also, it is clear that the illusion can occur with a wide variety of stimulus objects, including squares, circles, elipses, and irregular shapes. The Ames explanation is clearly not applicable to nontrapezoidal objects.

Graham $(1963,1965,1968)$ has examined the role of the motion parallax cue (differential angular velocity) in the illusion. He points out that this cue provides ambiguous depth information and suggests that in the face of this ambiguity the cue of linear perspective is responsible for the illusion.

It is the thesis of the present sudy that the illusion consists essentially in a nonveridical perception of depth, since the ordinal characteristics of the lateral component of motion are perceived correctly. Circular motion in a plane parallel to the line of sight cannot be perceived directly as can tangential motion across the field of vision, but must be mediated, consciously or unconsciously, by depth cues. Further, it is evident that in such illusions as the Ames trapezoid demonstration, there is not a mere lack of depth perception, but a confounding of it. A lack of depth perception would exist if the motion were seen as in a plane surface normal to the line of sight at an indeterminate distance. Under such circumstances an object would appear to change in contour. In the case of the trapezoid illusion, however, the long edge is seen as the nearer, both when it truly is and also when the short edge is nearer. During half of each rotation, then, the relative distance of parts of the object is perceived incorrectly.

In the Ames trapezoid demonstration, the cue of linear perspective has been manipulated so that it tends to provide false information about the relative distance of the parts of the trapezoid during part of its rotation. Linear perspective refers to the optical fact that an object of constant physical size casts a larger image on the retina when closer to the eye than when further away. In the absence of sufficient depth information from other sources there should be a tendency when viewing two objects or two parts of an object to see the smaller, nearer object or part as further away.

In the experiment reported here the cue of linear perspective was used without the possibility of support from shape connotation such as is present in the Ames trapezoid. Specifically, it was predicted that two luminous lines of unequal length, if rotated about a common parallel axis in a plane parallel to the line of sight, would induce illusory motion perception. The long line would frequently appear to oscillate back and forth, remaining closer to the viewer than the short line. The strength of the illusion was predicted to increase with increasing stimulus size disparity.

\section{METHOD}

The stimulus pairs illustrated in Fig. 1 were rotated at $33 \mathrm{rpm}$. Eight Ss viewed each pair both directly and $3 \mathrm{deg}$ peripherally (right), from $10 \mathrm{ft}$, for $90 \mathrm{sec}$, in a darkened room, and recorded each apparent reversal in direction of rotation via a response key electrically linked to a kymograph. Only the rotating lines, fluoresced under black light, were visable. A replicated $4 \times 4$ Latin square design was employed in ordering the presentation of stimuli.

\section{RESULTS}

The results are illustrated in Fig 2. There was, in general, an increased tendency for the illusion to occur with increased disparity in length of the line pairs. Peripheral viewing resulted in more illusory reversals than did direct viewing. Two Treatment (stimulus pairs) by Ss analyses of variance were applied, one for each viewing condition, and the treatment effect sum of squares was partitioned for orthogonal trend analysis (Edwards, 1960). For both viewing conditions a significant $(p<.01)$ linear component and a significant $(\mathrm{p}<.05)$ quadratic component were demonstrated. While strength of the illusion increased as a function of the disparity in the line pairs, at the extreme, with one line reduced to a point, a significant departure from a linear relationship was introduced.

\section{DISCUSSION}

The results suggest that sufficient formal similarity existed between line pairs to support a tendency toward size constancy and consequent misperception of the relative distance of the lines. Decrease of formal similarity with the lines and point (3:0) reduced the tendency for size constancy with consequent decrease in illusion strength.

The capacity of a rotated object to produce illusory reversals cannot be totally explained in terms of confounding depth cues. The two luminous lines of equal length $(3: 3)$, while they resulted in fewer illusory reversals than the other objects, did produce illusory reversals. With the reduction in effectiveness of true depth information such as occurs with peripheral viewing, or with monocular viewing, there are many internal and external factors, 

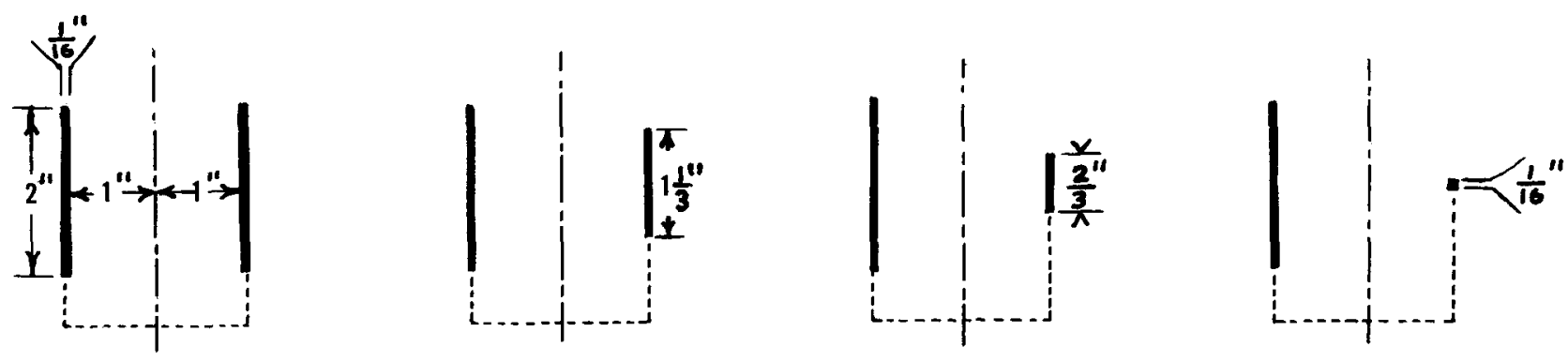

Fig. 1. The stimulus pairs used in the experiment. The middle dotted line indicates the axis of rotation. The stimulus line pairs provided length ratios of $3: 3,3: 2,3: 1$, and $3: 0$, covering the possible range of length disparity. These stimuli were produced by wire coated with luminescent paint to the dimensions indicated.

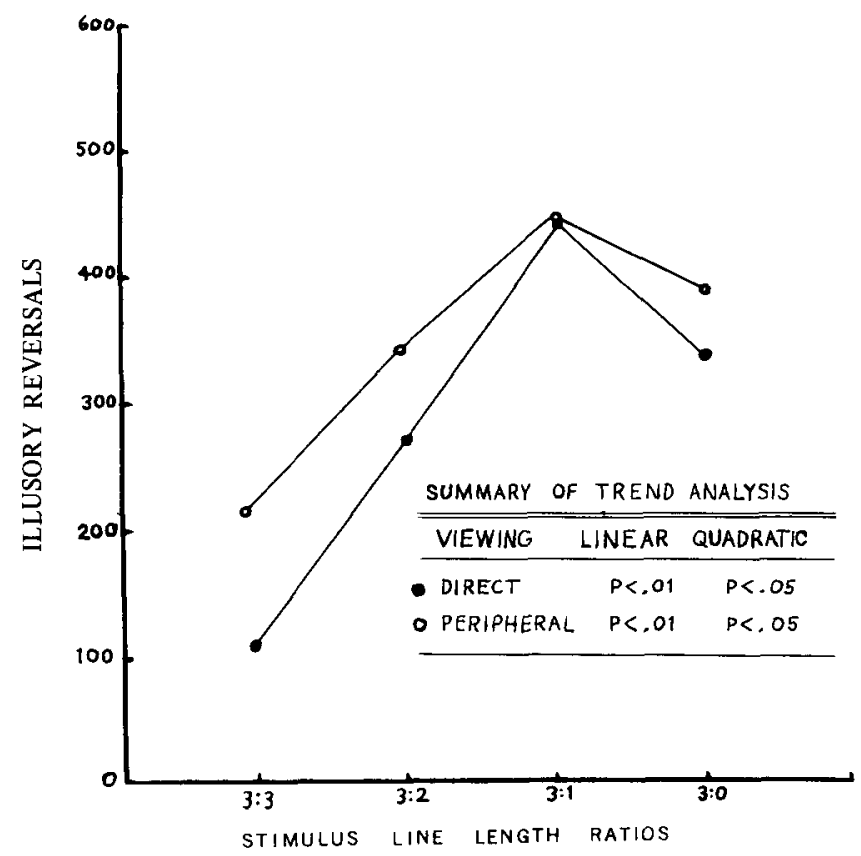

Fig. 2. Misperceived rotary motion as a function of stimulus size disparity.

in addition to confounding depth cues, that may lead to such nonveridical perception. This study was not designed for their identification or evaluation. Rather it was undertaken in the belief that the identification of confounding depth cues as significant illusion-producing factors represents a more parsi- monious, if only partial, explanation of these illusions than has previously been offered. It seeks to relate perceptual phenomena to stimulus variables before turning to such internal factors as assumptions of rectilinearity and also it represents an extension of some of the principles found useful in explaining visual space perception, namely the depth cues, to explanation in the realm of visual motion perception.

\section{REFERENCES}

ALLPORT, F. H. Theories of perception and the concept of structure. New York: Wiley, 1955.

AMES, A. Visual perception and the rotating trapezoidal window. Psychological Monographs, 1951, 65, No. 7 .

DAY, R. H., \& POWER, R. P. Frequency of apparent reversal of rotary motion in depth as a function of shape and pattern. Australian Journal of Psychology, 1963, 15, 162-174.

EDWARDS, A. L. Experimental design in psychological research. (Rev.). New York: Rinehart and Co., 1960.

GRAHAM. C. H. On some aspects of real and apparent visual movement. Journal of the Optical Society of America, 1963, 53, 1015-1025.

GRAHAM, C. H. Perception of movement. In C. H. Graham (Ed.), Vision and visual perception. New York: Wiley, 1965.

GRAHAM,C. H. Depth and movement. American Psychologist, 1968, 23, 18-26.

MULHOLLAND, T. B. Motion perceived while viewing rotating stimulus objects. American Journal of Psychology, 1956, 69, 96-99.

MURRAY, P. A. A quantitative study of the Ames oscillatory illusion. Dissertation Abstract, 1961, 22, 11.

PASTORE, N. Some remarks on the Ames oscillatory effect. Psychological Review, 1952, 59, 319-323.

ZEGERS, R. T. The reversal illusion of the Ames trapezoid. Transactions of the New York Academy of Sciences, 1964, 26, 377-400.

NOTE

1. Address: Department of Psychology, Saint Louis University, Saint Louis, Missouri 63103.

(Accepted for publication February 6, 1969.) 